\title{
Biosafety and Biosecurity Programme: Its Implementation, Requirements and Continuous Development at the Nanyang Technological University in Singapore
}

\author{
Tin Tun, Peter Rainer Preiser
}

School of Biological Sciences, Nanyang Technological University. Nanyang 639798, Singapore

\begin{abstract}
In Singapore, biosafety and biosecurity measures are controlled by the Biological Agents and Toxins Act (BATA) and other requirements by regulatory agencies. The law prohibits and otherwise regulates the possession, use, import, transhipment, transfer, and transportation of biological agents, inactivated biological agents, and toxins that are of public health concern. The law also defines the facility requirements for high risk biological agents and toxins. The containment facility (BSL 3) is a minimum requirement to handle biological agents that falls under Schedule 1 (Risk Group 3). The Nanyang Technological University School of Biological Sciences Biosafety Level 3 Facility (NTU-SBS BSL 3) was designed specifically for research involving potential hazardous biological materials. The facility requires yearly re-certification by an approved facility certifier to meet the local requirements and international biosafety standards for a containment facility in many instances. On the other hand, most NTU researchers conduct biological projects involving biological agents with low or moderate risk groups (Risk Groups 1 and 2 or biological agents described in schedule 3 and 4 of BATA) and GMOs, which need only a BSL 2 laboratory. BSL 2 laboratories are yet to be legally certified or registered in Singapore. Institutional Biosafety Committee (IBC) identifies the requirements; defines a minimum standard in the safe control of biological risks and registers all BSL 2 laboratories in the NTU. Therefore, under the guidance of the IBC, the University Biosafety and Biosecurity Programme includes the audit and certification program as a unique and an internal exercise to bring NTU biosafety to a higher level.
\end{abstract}

Key words: Biological Agents and Toxins Act, Biosafety Level, Institutional Biosafety Committee, Risk groups

\footnotetext{
This is an Open Access article distributed under the terms of the Creative Commons Attribution Non-Commercial License (http://creativecommons.org/licenses/by-nc/4.0) which permits unrestricted non-commercial use, distribution, and reproduction in any medium, provided the original work is properly cited.
}

Copyright (C) 2018 The Korean Society for Clinical Laboratory Science. All rights reserved.
Corresponding author: Tin Tun School of Biological Sciences, Nanyang Technological University, Nanyang Drive, Singapore 637551

Tel: 65-9753-5466

Fax:65-6791-3856

E-mail: tintun64@gmail.com

Received: May 29, 2018

Revised: June 5, 2018

Accepted: June 5, 2018

\section{INTRODUCTION}

The critical aspects of biosafety, biosecurity, and biocontainment have been in the spotlight in recent years. There have been increased international efforts to improve awareness of modern practices and concerns with regard to safety in life sciences research and to optimize frameworks, thereby minimizing risk of terrorist acquisition of deadly pathogens or accidental release of biological agents and their toxins.

Outbreaks of disease by emerging pathogens could pose significant challenges to global security by undermining national economies, international trade and travel, public health and safety. Any improper use of a biological agent and its toxin could have potentially devastating consequences on public health or the environment. An effective and comprehensive biosecurity to prevent unauthorized possession, loss, theft, misuse, diversion, or intentional release of biological agents and toxins is a shared responsibility at the international level since infectious disease is borderless. Today, genetic engineering advancement has empowered scientists to isolate any gene of interest from 
any organism or part thereof and incorporate it into a small self-replicating genetic element for multiplication as well as expression of the gene. The recombinant DNA molecule can be introduced into host cells such as bacteria, yeast and so forth to grow recombinant microorganisms with certain characteristics. Thus, scientists can manipulate genes and have they expressed in living organism to produce the desirable product. In fact, there is a possibility of incorporating unpredictable or unforeseen risks into those advancements. With the increasing number of countries adopting molecular tools and techniques in their life sciences research and development activities, the biosafety issues are gaining importance to ensure biological safety for the public and the environment.

There has been increasing awareness among the researchers, producers and users of genetically modified organisms (GMOs), administrators, policy makers, environmentalists and public about biosafety. Therefore, many countries have put into place regulatory policies and regulatory bodies for biomedical research and development of GMOs, however strict compliance to biosafety guidelines is still required.

\section{MATERIALS AND METHODS}

This study describes laboratory biosecurity planning for microbiological laboratories. Background of this study introduces the terms biosafety and biosecurity and looks at how they are used and what they mean in different settings. Previous agreements, understandings and proposals on biosafety and biosecurity, from past meetings of the convention, be investigated than respectively. Summary information on relevant activities undertaken by a number of international and regional organizations are included in Singapore. Sources of additional technical information are listed at the Nanyang Technological University in Singapore. This study provides an overview of biosecurity/ biosafety and the practices, equipment, and facilities for the safe and secure handling of dangerous pathogens in a laboratory setting.

\section{RESULTS}

\section{Biosafety and biosecurity awareness}

Biosafety refers to containment principles, technologies and the implementation of laboratory practices and procedures, specific construction features of laboratory facilities, safety equipment, and appropriate occupational health programs when working with potentially infectious microorganisms and other biological hazards. These measures are to reduce the exposure of laboratory personnel, the public and the environment to potentially infectious agents and other biological hazards. Laboratoryacquired infections (LAIs) have also started to receive more attention in recent years, in particular with regard to containment laboratory (biosafety level 3, or BSL 3) or maximum containment laboratory (biosafety level 4 , or BSL 4). LAIs may occur in research labs, clinical labs, or animal facilities, and from the community. There is a strong public health concern related to the LAIs, as an infected laboratory worker may transmit the infectious disease to his colleagues, family, or community at large. The term biosecurity is more complex as it can have different meanings in different contexts. Biosecurity refers to the mechanisms to establish and maintain security and oversight of pathogenic microorganisms, toxins and relevant resources. Laboratory biosecurity describes protection, control and accountability for valuable biological materials within the laboratory, in order to prevent their unauthorized access, loss, theft, misuse, diversion or intentional release [1]. While biosafety protects people from hazardous biomaterials, biosecurity protects such materials from people. Therefore, biosecurity concepts differ from biosafety concepts. The approaches used to achieve them are often similar or mutually reinforcing, but in some cases, they may have conflicts. For example, in the transportation of dangerous pathogens, biosafety recommends clear labelling of the material during transport, but from a biosecurity perspective, labelling of the material during transport may increase the risk of theft or misuse. 


\section{Singapore experiences}

In the last decade a number of emergent, or reemergent, diseases have reminded us of the constant threat posed by infectious diseases. These diseases affect humans, animals, plants, or multiple species, as recently demonstrated by the occurrence of diseases, such as Nipah virus outbreak, Hand Foot \& Mouth disease, Ebola outbreak, Severe Acute Respiratory Syndrome (SARS), Zika spread, avian influenza (H5N1), and the more recent influenza variant, H1N1. In each case, the rapid global spread of these diseases created enormous pressure on governments to diagnose, treat, and control the outbreaks. Singapore was affected by SARS outbreak in 2003 with 33 deaths and 238 cases (14\% fatality). Regarding LAIs, Singapore announced on 8 September 2003 that a post-doctoral worker in a SARS research lab in the National University of Singapore contracts the disease while working on the West Nile virus but recovered shortly thereafter. It was suspected that the two viruses mixed while he was doing his research. On 10 December 2003 a researcher in a SARS lab in Taiwan came down with SARS after returning from Singapore attending a medical conference, 74 people in Singapore were quarantined but none of them were infected (https://en.wikipedia.org/ wiki/Timeline_of_the_SARS_outbreak). The regional and global spread of emerging infectious diseases has driven Singapore to put emphasis on research in life sciences and to develop comprehensive system of control for potentially hazardous biological agents. The strict rules have been introduced to protect people from being exposed to the potentially hazardous biological agents and also to prevent terrorists from turning research samples into biological weapons. The laboratory environment needs to be defined in terms of biosafety and biosecurity capabilities.

\section{Biological Agents and Toxins Act (BATA)}

After SARS outbreak, as a legislative enforcement of biosafety and biosecurity issues, the Biological Agents and Toxins Act (BATA) came into force on 3 January 2006 in Singapore (Ministry of Health, 7 December 2005). The
BATA prohibits and otherwise regulates the possession, use, import, transhipment, transfer and transportation of biological agents, inactivated biological agents and toxins that are of public health concern [2]. Its objectives include preventing acts of bioterrorism, establishing a strong national biosafety culture and facilitating emerging bioscience industry in Singapore. The important objectives of the BATA are provision of safety practices in hand. The law also defines the facility requirements for high risk biological agents and toxins. The BATA adopts a schedule system for risk group classification different from WHO classification. Biological agents and toxins are classified into 5 different schedules depending on their risks. Containment facility (BSL 3) is a minimum requirement to handle biological agent which falls under schedule 1 (Risk Group 3). The schedule 2 (Risk Group 4) is to handle in a maximum containment facility (BSL 4). Biological agents or microorganisms with moderate or low risks fall under schedule 3 and schedule 4 (Risk Group 2) and are allowed to handle in a BSL 2 facility. To work with toxins listed in the schedule 5 requires a protected place under the Protected Areas and Protected Places Act [3]. A good regulatory framework will strengthen Singapore's standing as a biomedical hub and help it to attract world-class researchers. The laws have to strike a good balance between keeping research safe and being too restrictive. The requirements should not result in unnecessary cost increases, research being hampered, and scientists being discouraged from working to prevent disease outbreaks. There is a fine is of up to $\$ 1$ million Singapore dollars and life imprisonment if charges involve a deliberate attempt to use biological agents and toxins for biological warfare or any nonpeaceful purpose. Such severe penalties for convicted offenders reflect Singapore's serious commitment to biosafety and biosecurity [4].

\section{NTU-SBS BSL 3 facility}

The use of BSL 3 facilities is not limited to research and development, but is necessary for all aspects of laboratory work including clinical, diagnostic, teaching, and production facilities in which agents are stored and 
handled that may cause serious or potentially lethal diseases as a result of aerosol exposure. Nanyang Technological University School of Biological Sciences Biosafety Level 3 facility (NTU-SBS BSL 3) was specifically designed for research involving biological materials of potential hazard. It was one of pioneer groups of containment laboratories in Singapore. Thus, NTU Biosafety has started at the School of Biological Sciences. An annual inspection and audit, and preventive maintenance of the laboratory is carried out to ensure all aspects of the integrity and safety features of the containment facility. BSL 3 laboratory needs to have negatively pressurised condition and directional airflow for the containment purpose of all possible risks inside. Negative pressure within the facility is achieved by supplying less air into the room than the volume of air exhausted via the biological safety cabinet and room general exhausts. A dedicated control system continuously monitors and maintains the pre-set flow rate difference between the supply and exhaust air to achieve the required room pressurization. The supply air is filtered at the Air Handling Unit to extend the life-span of the exhaust high-efficiency particulate air (HEPA) filters. All air exhausted from the laboratory is filtered through HEPA filters [5]. The adopted comprehensive approach to biosafety is based on a combination of administrative controls, safety practices and procedures, engineering controls, and personal protective equipment. As the facility was designed to accommodate multipurposeusers with regular staff training and turnover; thus, user training plays a critical role. In spite of safety programs in a laboratory, accidents still happen. Biological spills tend to be the most common accidents. Good safety programs, training, and serious precautions can help reduce the number, frequency, and severity of accidents. All possible scenarios including the worst cases that could happen in the laboratory are identified and stressed in the training program. Training contents address related legislation, regulations, guidelines and facility requirements; bio safety culture and concepts; standard procedures and laboratory practices; laboratory instruments and their maintenance; emergency responses; administrative requirements, etc [6]. Emergency procedures detail various preventive measures and operational actions to be taken when an emergency occurs at the BSL 3 facility. The NTU-SBS BSL 3 facility developed an emergency response plan (ERP) comprised of standard operating procedures for various emergencies [7]. The plan consists of many parts that provide useful information for all identified potential emergency situations. On a regular basis, emergency drill exercise is conducted under the guidance of Singapore Civil Defence Force (SCDF) [8]. Facility management conducts a biosecurity drill or red teaming exercise as instructed by the Singapore Police Force (SPF) and the Ministry of Health (MOH) [9]. As a containment facility requirement, the facility is certified by a $\mathrm{MOH}$-approved facility certifier (AFC) after thorough checking all structural or physical conditions under engineering aspects, administrative control measures, biosafety procedures and practices and all documents and records. The facility needs re-certification yearly or upon any design or structural change made to the facility. Having met the local requirements and international biosafety standards for a containment facility in many instances, the NTU-SBS BSL 3 facility was in operation and first registered with the $\mathrm{MOH}$ as a certified facility in 2004. Keeping the facility operational is indeed an expensive exercise in term of budget, time and efforts. Recertification process has continued for 8 continuous years till 2012. Later, the facility stopped re-certification since university research projects rarely involved handling of biological agents with high risks (schedule 1 or risk group 3) in other words there were no potential users for the BSL 3 laboratory. Now the facility has been shut down and the building itself is being under major renovation. Whether or not keeping a containment facility will be up to the university management.

\section{Institutional Biosafety Committee (IBC)}

It is a BATA requirement for a BSL3 facility to establish an Institutional Biosafety Committee (IBC) which includes at least biosafety coordinator, microbiologist, facility main- 
tenance personnel and representative from senior management. First 6 member IBC was established specifically for the management of the NTU-SBS BSL 3 facility in 2004. The committee was led by the SBS Chair and all IBC members were from the school. The IBC is responsible to formulate and review biosafety policies and programs including training of staff, conducting risk assessment, reviewing and approving the research projects. For project involving genetic manipulation work, the IBC liaises with Genetically Modified Advisory Committee (GMAC) for its review and approval. Government authorities - MOH and Agri-Food and Veterinary Authority (AVA) are communicated as required [10, 11]. In early days, the SBS was a major research facility or center where biological research projects were mostly carried out. Later, other schools and research centers, School of Chemical and Biomedical Engineering, Nanyang Environment and Water Research Institute, Singapore Centre for Environmental Life Sciences Engineering and Lee Kong Chiang School of Medicine increase to conduct biological research projects. New IBC is more university-wide and comprises members including faculty or academic staff and non- academic staff from various schools and research centers in the university. The IBC develops the "University Biosafety and Biosecurity Programme" and looks into biosafety issues in all aspects. It takes bigger roles and responsibilities such as compliance issues, review and assessment of biological research projects, BSL 2 laboratory audit and certification program, biosafety training requirements, registering all biological research projects, etc. NTU researchers commonly use biological agents that fall under schedule 3 and schedule 4 or risk group 2; handling of those low or moderate risk microorganisms is allowed in a BSL 2 laboratory. There are many such laboratories in NTU schools and research centers. The IBC emphasises on management of BSL 2 environment and the importance of BSL 2 facilities and practices are highlighted in the biosafety and biosecurity program. Biosafety audits, routine laboratory inspections and unscheduled checks for any biological work are conducted to ensure all the risks are adequately addressed and additionally seeks to assure the senior management that all foreseeable risks are taken under control. All biological project proposals are reviewed by the IBC and are registered with the Biological Project Number (BPN) application system. BPN Administrators are appointed at schools or research centers to monitor biological projects and to facilitate the administrative process in biosafety and biosecurity program.

\section{BPN online registration}

BPN Online Registration System is in the process of development as a part of university biosafety and biosecurity program. Once the system is in operation, every step in the process will be done online for example, BPN submission by researchers, IBC review and approval, GMAC referral process and approval, program report generation and documentation, etc.

\section{Safety manual for biological work}

A comprehensive Safety Manual for Biological Work is developed and approved by the IBC. It provides university-wide biosafety measures for the use and manipulation of biological agents, as well as the containment procedures and control of biohazards. Roles and responsibilities of IBC, principal Investigator and researchers, administrators, etc, biosafety requirements, managing biosafety, laboratory procedures and practices and BPN application requirements are addressed in brief. The manual is readily available on the university website for all NTU laboratory users.

\section{Register for BSL 2 laboratories}

BSL 2 laboratories are allowed to use for biological projects involving biological agents with low or moderate risk groups (Risk Groups 1 and 2 or biological agents described in schedule 3 and 4 of BATA) and GMOs. But, these BSL 2 laboratories are yet to be legally certified or registered in Singapore. It is known that the ministries ( $\mathrm{MOH}$ and/or the Ministry of Manpower) are looking into biosafety issues for BSL 2 laboratories but no specific requirements have been defined so far [12]. However, 
these research laboratories involve not only biological risk; there are other associated risks such as radiation, chemical, physical and mechanical nature [13]. Biological risk is generally more complicated to handle as microorganisms exhibit pathogenic and virulence characteristics. Thus, under the guidance of the IBC, the BSL 2 audit and certification program is implemented as a unique and an internal exercise to bring the NTU biosafety up to a higher level [14]. Having met the requirements and set criteria, the inspected or audited NTU laboratory is registered as a BSL 2 laboratory.

\section{BSL 2 certification criteria}

The "BSL 2 Certification Criteria" document has been developed and approved by the IBC for strictly internal use to identify requirements, which defines a minimum standard in safe control of biological risks in a BSL 2 laboratory in the NTU. The document is condensed from recommendations laid down in WHO Biosafety Manual [15], Biosafety in Microbiological and Biomedical Laboratories (NIH-CDC, 2009) [16] and legal requirements in Singapore. This document is available for the NTU laboratory users as a shared document on the webpage. Laboratory certification is the systematic review of all safety features and processes associated with the laboratory. This include assessment of a) engineering controls such as biological safety cabinets and ventilation; b) administrative control such as work risk assessments, biological risk review, standard operating procedures (SOP), documentation and record retention systems; and c) use of personal protective equipment. Standardization and regular certification provides accountability and ensures proper implementation of biosafety controls in accordance to acceptable practices and risk managements.

\section{User's competency in biosafety}

Training for BSL 3 staff is mandated under the Biological Agents and Toxins Act (BATA), which has been in force in Singapore since 2006 [2]. The MOH also releases circulars or instructions regarding BSL 3 issues whenever required. Every facility appoints a biosafety coordinator who participates in the structured biosafety training course conducted by a $\mathrm{MOH}$-approved training provider (ATP) and who must pass the competency test administered by the $\mathrm{MOH}$. The biosafety coordinator acquires experience in the area of biosafety management and undergoes such training as the Director of Medical Services requires from time-to-time. He or she must be a member of the institutional biosafety committee that formulates safety measures, programs, codes of practice, and all policies including training of staff. The in-house training program was approved by the IBC to train potential users of the NTU-SBS BSL 3 laboratory [6]. Program procedures consist of lectures, practical demonstrations and simulations, video learning, discussions, reviews and evaluation of training, and documentation. Training contents address related legislation, regulations, guidelines and facility requirements; biosafety culture and concepts; standard procedures and laboratory practices; laboratory instruments and their maintenance; emergency responses; administrative requirements; and application procedures or protocols adopted at the facility. Exercises or simulated situations are conducted to provide hands-on experience for accidental procedures such as biological spill management, medical emergencies, and fire emergencies. Trainees get precise and correct information about what to do, whom to contact, what equipment is available for response, what steps to follow, and how safe evacuations take place during particular emergencies [7]. User's competency in biosafety is very important for safe handling of biological agents and biological materials which can cause laboratory acquired infection (LAI). It is compulsory for all NTU laboratory users to take the "Online Basic Biosafety Training Programme" which is available on the NTULean at the university website. The online course includes biosafety concepts, legislation requirements, good microbiological practices (GMP) and emergency procedures and/or spill management. After reviewing the program, participant needs to answer the questions; $70 \%$ score is required to complete the test with a pass. System records and maintains a list of successful participants. It is noted that introduction of a standardized 
biosafety training course at national level under Workforce Development Agency (WDA) program by the Ministry of Manpower is in process. The strength of this program is to standardize or harmonize practices in this area and to promote mutual recognition of competency of laboratory users from various institutions - hospitals, universities, polytechnics, research centers and other industries. When the program is ready and publicized, employers have to send their staff to the agency-approved training provider or training institute. "Biosafety Passport" will be awarded upon completion of the course. Holder of the passport will be well qualified and recognized as a competent worker in any biological laboratory in Singapore.

\section{DISCUSSION}

NTU provides basic equipment and facility requirements for biological research works at BSL 2 laboratories. Laboratory facility is mostly adequate and sufficient for safe handling of biological agents with low or moderate risk provided that proper maintenance of equipment and good microbiological practices are in place. There is a constant need to train our researchers or laboratory users and to nurture the safety culture in biological and biomedical research to be vigilant and adhere to good microbiological practices. We put so much effort to raise commitment toward biosafety and biosecurity concerns for laboratory users at multiple levels. Individual schools/centers, teams and research groups have taken it upon themselves to implement biosafety practices, but this is to be done in a consistent manner. Integrated safety culture and a standardized biosafety practices is to establish. This is an expectation of the program and all persons from the top to the bottom are accountable.

\section{Acknowledgements: Sincere appreciations go to:}

- IBC members: for their support to the programme

- Safety Officers (Office of Health, Safety and Emergency): for coordinating with schools and research centres; help for development of the programme in many ways

- BPN Administrators at schools and research centres: for their inputs and assistance in the programme Conflict of interest: None

\section{REFERENCES}

1. WHO. Biorisk management, Laboratory biosecurity guidance. Geneva: WHO/CDS/EPR; 2006. p1-33.

2. Singapore Statutes Online. Biological Agents and Toxins Act 36 of 2005 [internet]. Singapore: Government of Singapore; 2006 [cited 2018 May 20]. Available from: http:// statutes. agc.gov.sg/.

3. Singapore Ministry of Health. Updated Biological Agents and Toxins List [internet]. Singapore: Singapore Ministry of Health; 2016. [cited 2018 May 20]. Available from: https://www.moh. gov.sg/content/dam/moh_web/BioSafety/docs/List_of_ Biological_Agents_and_Toxins.pdf.

4. Tun T, Sadler KE, Tam JP. Biological agents and toxins act: Development and enforcement of biosafety and biosecurity in Singapore. Appl Biosaf. 2007;12:39-43.

5. Tun T, Sadler KE, Tam JP. Implementation of a biosafety level 3 (BSL-3) Facility in Singapore: Requirements, work practices, and procedures. Appl Biosaf. 2006;11:15-23.

6. Tun T, Sai-Kit AL, Sugrue RJ. In-house BSL-3 user training: Development and implementation of programme at the Nanyang technological university in Singapore. Appl Biosaf. 2009;14:89-97.

7. Tun T, Sadler KE, Tam JP. A novel approach for development and implementation of an emergency response plan for the BSL-3 laboratory service in Singapore. Appl Biosaf. 2008;13: 158-163.

8. Singapore Civil Defense Force. Guidelines for company emergency response plan [internet]. Singapore: Singapore Civil Defense Force; 2007. [cited 2018 May 20]. Available from: www.scdf.gov.sg.

9. Singapore Ministry of Health. Commencement of Biological Agents and Toxins Act. MOH Circular, 70(13/15-0026) [Internet]. Singapore: Singapore Ministry of Health; 2005 [cited 2018 May 20]. Available from: https://www.moh.gov.sg/.

10. Singapore Agri-Food Veterinary Authority. Requirements for the import and transshipment of pathogens. Circular IED 2/1 [Internet]. Singapore: Singapore Agri-Food Veterinary Authority; 2005 [cited 2018 May 20]. Available from: https:// www.ava. gov.sg/.

11. Genetic Modification Advisory Committee. The Singapore biosafety guidelines for research on the genetically modified organisms. Singapore: Genetic Modification Advisory Committee [internet]. 2006. [cited 2018 May 20]. Available from: https:// www.gmac.gov.sg.

12. Singapore Ministry of Manpower. "Workplace Safety and Health Act" [internet]. Singapore: Ministry of Manpower; 2006 [cited 2018 May 20]. Available from: http://www.mom.gov.sg/workplace- safety-and-health/workplace-safety-and-health-act.

13. Singapore Ministry of Manpower. "Workplace Safety and Health (Risk Management). Regulations" [internet]. Singapore: Ministry of Manpower ; 2006 [cited 2018 May 20]. Available from: http:// 
www.mom.gov.sg/workplace-safety-and-health/safety-and-h ealth-management-systems/risk-management.

14. Tin T, Lee KW. BSL2 audit and certification program: An effort to harmonize and to raise standards in both laboratory infrastructure and biosafety practices in Singapore. Biomed Sci Letters. 2016;22:65-74. http://dx.doi.org/10.15616/BSL.2016. 22.3.65.

15. World Health Organization. Laboratory biosafety manual 3rd ed
[Internet]. Geneva: World Health Organization; 2004 [cited 2018 May 2]. Available from: http:// www.who.int/csr/ resources/publicatios/biosafety/WHO_CDS_CSR-LYO2004_ 11/en/Biological.

16. Centers for Disease Control and Prevention, National Institute of Health. Biosafety in microbiological and biomedical laboratories. 5rd ed. Atlanta: Centers for Disease Control and Prevention; 2009. 